\title{
Operation of a traction machine's wheel mover with a locked asymmetrical balance bar
}

\author{
Vladimir Zhulay ${ }^{1 *}$, Yuriy Ustinov ${ }^{1}$, Vitaliy Tyunin $^{1}$, and Aleksey Shchienko ${ }^{1}$ \\ ${ }^{1}$ Voronezh State Technical University, 394006, Voronezh, st. 20th Anniversary of October, 84, Russia
}

\begin{abstract}
The problems of determining the parameters of a traction machine's wheel mover with a locked asymmetrical balance bar operation, taking into account the kinematic mismatch arising from the redistribution of the vertical reactions at the driving wheels due to off-center pushing force, have been considered. An algorithm for calculating traction and braking forces, as well as drive wheels' slippage and skid of an asymmetric balance bar based on their traction balance and the condition of equal actual speeds under a variable vertical load, have been presented. As a result of research it has been found that the greatest additional loads in the drive and tire wear of the wheel mover with a locked asymmetric balance bar will occur when it moves in transport mode on a support surface with high traction properties.
\end{abstract}

A locked drive of the drive wheels located on the balance bar is often used in drivelines of wheeled traction machines that operate under heavy loads, [1]. Such a scheme makes it possible to simplify the driveline design and provide high maneuverability and developed traction force, especially on supporting surfaces with weak and uneven traction and coupling properties. It also provides good wheel contact when driving on uneven surfaces.

Therefore, the driving wheels balanced suspension is widely used in various transport and traction technological machines.

But it has some drawbacks. The main one is the redistribution of vertical reactions to the balance bar driving wheels $[2,3]$. This is especially evident in traction machines that have a balance bar with an eccentric. Due to its presence, the pushing force in the suspension joint of the balance bar causes a torque that increases the load on the rear driving wheels and decreases on the front ones $[2,3]$. This, in turn, leads to a change in the wheels' power radii and, as a result, to the appearance of kinematic mismatch, an increase in loads in the wheel drive and a decrease in the efficiency of the wheel engine due to additional skidding or slipping, which occur when the driving wheels' rolling radii are aligned [2-4].

Various design solutions have been proposed to eliminate this drawback. In [2] the grader balance bar design has been substantiated. The design ensures the equality of normal reactions to the balance bar's drive wheels regardless of the developed traction force magnitude. To do this, the balance bar must not have an eccentricity, and the gear ratio of its mechanical gears must be equal to $1 / \eta_{\sigma}$, where $\eta_{\sigma}$ is the efficiency of the mechanical

\footnotetext{
*Corresponding author: zhulai@vgasu.vrn.ru
} 
gears. But in the absence of eccentricity, the clearance decreases, and, consequently, the technological machines' passability on undeveloped sites and roads decreases.

In [3] it is proposed to equalize the loads on the drive wheels of the balance bar with eccentricity by changing the leverage ratio of the drive wheels' position. This design of socalled asymmetrical balance bar ensures an increase in the distance between the rear wheel to the axis of suspension to the frame and a corresponding decrease in this distance for the front wheel. The magnitude of the resulting torque from the difference in the normal reactions leverage of the bearing surface to the drive wheels should be equal to the torque from the pushing force in the balancer bar joint and its eccentricity at a certain operating mode.

For traction technological machines this mode is taken as the maximum traction power mode [2-4]. In other operating modes the difference in normal reactions to the driving wheels of the balance bar will be present.

In $[5,6]$ the issues of reactions redistribution to the right and left sides of the rear drive axle and related power losses in the ball joints of other transmission elements are considered. For technological machines with rigid massive rear drive axles these phenomena will be insignificant.

Thus, the main modern design solution for equalizing the loads on the driving balance bar's wheels with the eccentricity of traction machines is to change the ratio of the wheel mounting leverages, i.e. the use of an asymmetric balance bar.

As noted above, this design ensures equal loads on the driving wheels only in one mode of the machine traction loading. And when the machine is moving in transport mode, the difference in normal reactions to the wheels will be significant, therefore, the difference in the power radii of the driving wheels will also be significant.

The operation of a blocked wheel mover of a car with different driving wheels' power radii is considered in detail in the academician E.A. Chudakov works [7].

He assumes that ensuring the equality of the actual driving wheels' speeds is due to the tangential elasticity of their tires. The alignment of the free rolling radii $r_{k 1}$ and $r_{\kappa 2}$ is determined from the equations

$$
\begin{array}{r}
r_{\kappa 1}=r_{c 1}-\lambda P_{1}=r_{\kappa 2}=r_{c 2}-\lambda P_{2}, \\
\text { or } \quad P_{1}-P_{2}=\frac{r_{c 1}-r_{c 2}}{\lambda}=\frac{c}{\lambda},
\end{array}
$$

where $r_{c 1}$ and $r_{c 2}$ are the power radii of the wheels 1 and 2 of the driving nondifferential axis;

$P_{1}$ and $P_{2}$ are the total circumferential forces of the wheels 1 and 2;

$\lambda$ is the coefficient of the tires' tangential elasticity;

$c$ is the coefficient equal to $c=r_{c 1}-r_{c 2}$.

With a total circumferential force equal to $P=P_{1}+P_{2}=0$ (uniform movement without traction load), we have

$$
P_{1}=0,5 \frac{c}{\lambda} ; P_{2}=-0,5 \frac{c}{\lambda},
$$

i.e. the circumferential force $P_{1}$ of the larger wheel (the wheel with more force radius $r_{c 1}$ ) will be the traction force, and the circumferential force $P_{2}$ of the lower wheel will be the braking force [7].

It is also noted that as the total circumferential force $P$ increases from zero, the modulus of the circumferential force $P_{2}$ decreases, reaches zero, and with further growth of $P$, the smaller wheel 2 becomes the leading one. 
The process of technological machines' interaction with the support surface of the wheel drive significantly differs from the working conditions of cars. They equalize the translational (actual) speeds of driving wheels with a blocked drive by skidding or sliding $[2,4]$.

The equality of the actual speeds $V_{d 1}$ and $V_{d 2}$ of the driving wheels with a blocked drive of traction technological machines is described by the equation $[2,4]$

$$
V_{d 1}=V_{d 2} \text { or } V_{T 1}\left(1-\delta_{1}\right)=V_{T 2}\left(1-\delta_{2}\right)
$$

Where $V_{T 1}$ and $V_{T 2}$ are the theoretical circumferential speeds of the drive wheels.

The theoretical wheel speeds are equal to $[2,4]$

$$
V_{T 1}=\omega_{\kappa} r_{c 1} ; V_{T 2}=\omega_{\kappa} r_{c 2},
$$

where $\omega_{\kappa}$ is the angular speed of the driving wheels.

The magnitude of the power radius of a wheel with a pneumatic tire depends on its size and the vertical load acting on it $[2,4]$

$$
r_{c}=r_{0}-\frac{R}{E_{u}},
$$

where $r_{0}$ is the free radius of a wheel with a pneumatic tire;

$R$ is the vertical load (normal reaction of the support surface) on the wheel;

$E_{u}$ is the radial stiffness of the wheel with a pneumatic tire.

The slippage value $\delta$ of each drive wheel also depends on the vertical load $R$, as well as on the traction force (longitudinal traction force) of the wheel mover $T_{\kappa}$ and on the supporting surface type, which determines the form of the function $f$ depending on $[2,4]$

$$
\delta=f\left(\frac{T_{\kappa}}{R}\right) .
$$

$\frac{T_{\kappa}}{R}=\psi$ is the relative traction force.

Thus, in contrast to transport vehicles, for traction machines, to solve the problem of driving wheels with a blocked drive having kinematic misalignment it is necessary to take into account the variability of the vertical load on them, the power radii depending on this load and the skidding of each wheel.

Let's consider the solution of the problem of the wheel drive with a blocked drive of an asymmetric balance bar of a traction machine parameters determining, the design scheme of which is shown in Fig. 1.

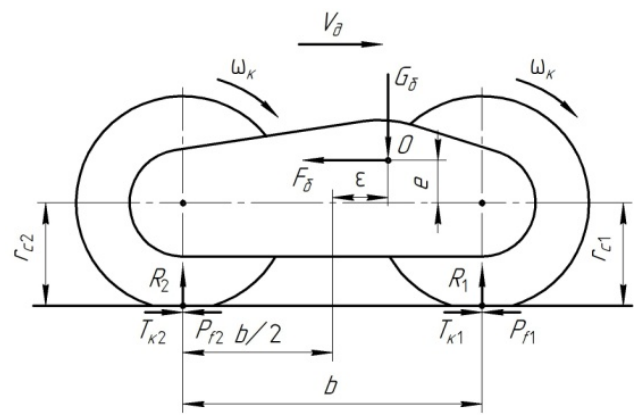

Fig. 1. The design scheme of an asymmetric balancing drive of a traction machine. 
The following notation is used in the design diagram: $G_{\sigma}$ is the gravity of the motor grader transmitted to the hinge (suspension axis) of the balance bar, taking into account its weight with the wheels; $F_{\sigma}$ is the horizontal reaction (traction resistance of the machine) in the hinge of the balance bar; $T_{\kappa 1}$ and $T_{\kappa 2}$ are the traction (braking) forces of the drive wheels; $R_{1}$ and $R_{2}$ are the normal reactions of the bearing surface to the drive wheels; $r_{c 1}$ and $r_{c 2}$ are the power radii of the drive wheels; e is the eccentricity of the balancer; $b$ is the base of the balance bar; $\varepsilon$ are the displacements of the balance bar's suspension axis; $P_{f 1}=$ $f_{k 1} R_{1}$ and $P_{f 2}=f_{k 2} R_{2}$ are the rolling resistance forces of the drive wheels, where $f_{k 1}$ and $f_{k 2}$ are the rolling resistance coefficients.

When solving this problem, the following assumptions are made.

The blocked balance drive is equipped with the wheels with pneumatic tires of the same size and the same internal air pressure in them. Therefore:

$$
r_{01}=r_{02}=r_{0} ; E_{u 1}=E_{u 2}=E_{u} ; f_{k 1}=f_{k 2}=f_{k} ; \omega_{k 1}=\omega_{k 2}=\omega_{\kappa}
$$

The initial linear sections of the dependences of slipping $\delta$ and skidding $(-\delta)$ on the relative thrust $\psi$ are considered which are identical [2, 4]

$$
\delta_{1}=k_{\delta}\left(\psi_{1}\right) ; \delta_{2}=k_{\delta}\left(\psi_{2}\right) ;-\delta_{1}=k_{\delta}\left(-\psi_{1}\right) ;-\delta_{2}=k_{\delta}\left(-\psi_{2}\right),
$$

where $k_{\delta}$ is the coefficient of proportionality.

To solve this problem, let's start with determining the parameters of the wheel mover with a blocked drive of the asymmetric balance bar of the traction machine when it is moving in transport mode, $F_{\sigma}=0$.

Ensuring the condition (1) of equality of the driving wheels' actual speeds and the difference in their power radii caused by different values of the vertical loads acting on them will be carried out by slipping and skidding of these wheels.

For loading conditions of the asymmetric balance bar shown in Fig. 1, when $R_{1}>R_{2}$ and, therefore, $r_{c 1}<r_{c 2}$, the difference in the power radii of the wheels is

$$
\Delta r_{c}=r_{c 1}-r_{c 2}
$$

Let's write the condition (4) taking into account the expression (5) and the equality of angular velocities (8) in the form $r_{k 1}=r_{\kappa 2}$, or

$$
r_{\kappa 1}=r_{c 1}\left(1-\delta_{1}\right)=r_{c 1}-\Delta r_{1} ; r_{\kappa 2}=r_{c 2}\left(1-\delta_{2}\right)=r_{c 2}-\Delta r_{2},
$$

where $\Delta r_{1}=r_{c 1} \delta_{1}$ is the share of difference $\Delta r_{c}$ compensated by wheel skidding 1 (because of skidding we use $(-\delta))$;

$\Delta r_{2}=r_{c 2} \delta_{2}$ is the share of difference $\Delta r_{c}$ compensated by wheel slipping 2 .

The values of shares $\Delta r_{1}$ and $\Delta r_{2}$ are determined from the condition of their proportionality to the values $r_{c 1}$ and $r_{c 2}$ according to the equation (11).

The values of skidding $\left(-\delta_{1}\right)$ and slipping $\delta_{2}$ are calculated according to $\Delta r_{1}$ and $\Delta r_{2}$

$$
-\delta_{1}=\frac{\Delta r_{1}}{r_{c 1}} ; \delta_{2}=\frac{\Delta r_{2}}{r_{c 2}}
$$

Knowing the values of skidding and slipping of the driving wheels from formulas (9), it is possible to determine the magnitude of their braking and traction

$$
T_{\kappa 1}=-\psi_{1} R_{1}=R_{1} \frac{-\delta_{1}}{k_{\delta}} ; T_{\kappa 2}=\psi_{2} R_{2}=R_{2} \frac{\delta_{2}}{k_{\delta}}
$$


In addition to the condition of equality of the drive wheels' actual speeds with a uniform movement of the balancer without traction load, it is also required that the condition $F_{\sigma}=0$ be met, i.e. traction and braking forces of the wheels to be balanced. The conditions for fulfilling this equality will depend on the operating modes of the balance bar wheels.

In our case, when $r_{c 1}<r_{c 2}$, the first wheel will be braking, and the second one will driving $[4,7]$. Then the traction force of the driving wheel $T_{k 2}$ will be balanced by the resistance to the braking wheel movement 1 , equal to the sum of the resistances $\left(T_{\kappa 1}+P_{f 1}\right)$ $[2,4,7]$. Denote $T_{K I}=\left(T_{\kappa 1}+P_{f 1}\right)$.

The calculation of the algorithm for determining the parameters of the operation of the blocked wheel mover of the asymmetric balance bar of the traction machine based on the above equations (4) - (13) and conditions is carried out using Mathcad engineering software for the following initial data $[2,8]$ in accordance with the calculation scheme adoption (fig. 1). $r_{0}=0,56 \mathrm{~m} ; b=1,6 \mathrm{~m} ; e=0,1 \mathrm{~m} ; \varepsilon=0,25 \mathrm{~m} ; G_{6}=80 \mathrm{kN} ; E_{u}=640 \mathrm{kN} / \mathrm{m} ; k_{\delta}=0,1 ; f_{k}=$ 0,05 .

For these conditions, the dependences of the normal reactions $R_{1}$ and $R_{2}$ on the drive wheels on the relative horizontal reaction in the balance bar hinge $\psi=\frac{F_{\sigma}}{G_{\sigma}}$ have the form

$$
R_{1}=52,5-24,8 \psi, \mathrm{kN} ; R_{2}=27,5+24,8 \psi, \mathrm{kN}
$$

As a result of calculations to meet the condition $V_{d 1}=V_{d 2}$ with $\psi=0$ we have: $r_{c 1}=0,514 \mathrm{~m} ; r_{c 2}=0,536 \mathrm{~m} ; \delta_{1 V}=-0,0217 ; \delta_{2 V}=0,02 ; r_{\kappa 1}=0,525 \mathrm{~m} ; r_{k 2}=0,525 \mathrm{~m}$; $T_{K I}=-8,78 \mathrm{kN} ; T_{K 2}=5,49 \mathrm{kN}$.

$\delta_{V 1}$ and $\delta_{V 2}$ are the values of slipping and skidding required to ensure the condition $V_{d 1}=V_{d 2}$.

The calculation results show that the condition $F_{\sigma}=0$ is not satisfied because the value of the braking force $T_{K I}$ is greater than the traction force $T_{\kappa 1}$.

To fulfill this condition, it is necessary that the lagging wheel 1 has less braking force, and the run-down wheel develops greater traction.

The value of these forces difference is equal to

$$
\Delta T_{\kappa}=\left|T_{K I}\right|-\left|T_{\kappa 2}\right|=8,78-5,49=3,29 \mathrm{kN} .
$$

The creation of additional traction will cause an increase in wheel slip by the value $\Delta \delta_{T}$ $[4,9]$. An increase in the slipping of the balance bar's driving wheels, which will provide the compensation for the difference in the efforts $\Delta T_{\kappa}$, will be

$$
\Delta \delta_{T}=\frac{\Delta T_{\kappa} k_{\delta}}{G_{\sigma}}=\frac{3,29 \cdot 0,1}{80}=0,0041 \text {. }
$$

The calculation of the values of the locked wheel mover balance bar parameters operation, ensuring the fulfillment of the conditions of the actual speeds of movement equality, as well as traction and braking forces of the wheel mover has the following results: $r_{c 1}=0,514 \mathrm{~m} ; r_{c 2}=0,536 \mathrm{~m} ; \delta_{10}=\delta_{1 V}+\delta_{1 T}=-0,018 ; \delta_{20}=\delta_{2 V}+\delta_{2 T}=0,024$; $r_{\kappa 1}=0,523 \mathrm{~m} ; r_{\kappa 2}=0,523 \mathrm{~m} ; T_{K I}=-6,62 \mathrm{kN} ; T_{\kappa 2}=6,62 \mathrm{kN}$.

Thus, in traction machines, the difference in the power radii of the driving wheels with a blocked drive is equalized due to these wheels' slipping and skidding. The values of this process consist of the running wheel skidding of the and the skidding of the lagging wheel and the slipping which provides a balance of traction and braking forces.

The values of the operation parameters made using the Mathcad engineering software according to the above algorithm are presented in Fig. 2. 


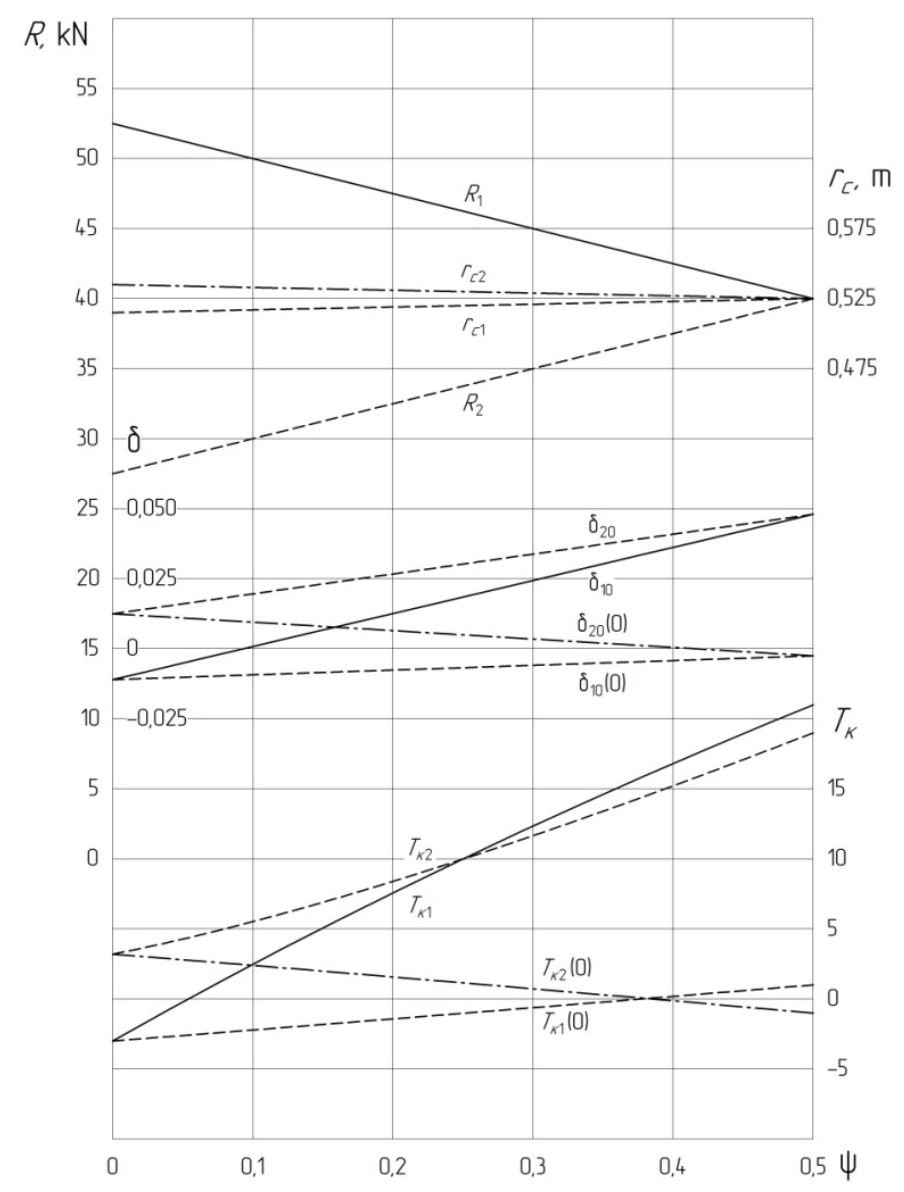

Fig. 2. The dependences of the values of the parameters of the work of a wheel mover with a locked drive of an asymmetric balance bar on its relative traction force.

For the convenience of analysis, the graph shows the lines of the values of the slipping (skidding) components $\delta_{20}(0), \delta_{10}(0)$ ensuring alignment of the rolling radii of the wheels $r_{\kappa 1}, r_{k 2}$ and the components of traction (braking) $T_{\kappa 2}(0), T_{K I}(0)$.

From the analysis of the graphical dependencies presented, it can be seen that the difference in the power radii $r_{c 1}$ and $r_{c 2}$ caused by the redistribution of the vertical loads $R_{1}$ and $R_{2}$ on the balance bar's driving wheels has a significant effect on the power load of the wheel mover of an asymmetric balance bar with a locked drive in the zone of small values of traction forces.

Consequently, when driving a vehicle with an asymmetrical balance bar, in its locked drive significant additional loads will occur due to slipping and skidding of the drive wheels, as well as increased tires wear.

Due to the fact that the vehicles movement in the transport mode is carried out mainly on flat surfaces with high traction and coupling qualities, we consider the effect the supporting surface on the load of the wheel mover with a locked drive of an asymmetric balance bar.

The traction and coupling qualities of the support surface influence the form of the dependence $\delta=f(\psi)$, which is determined for our assumptions by the value of the coefficient $k_{\delta}$. For asphalt concrete $k_{\delta} \approx 0,01$, and for loose soil $k_{\delta} \approx 0,2[2,4,7,9,10]$. 
The results of determining the dependences of the operation parameters of the wheel mover of an asymmetric balance bar with a blocked drive on the support surface properties are shown in figures 3 and 4 .

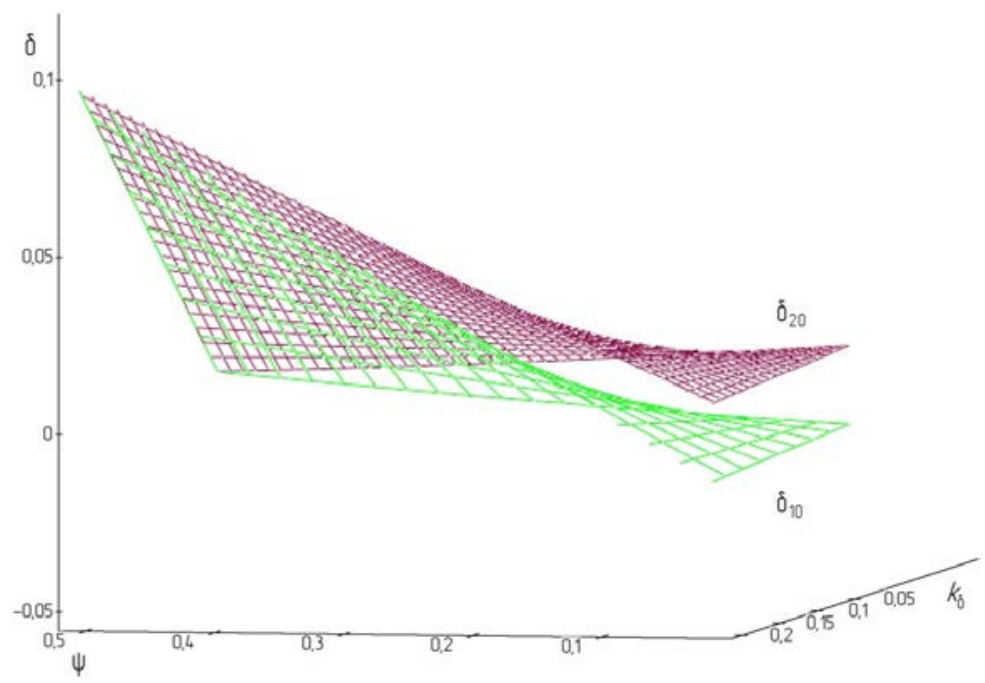

Fig. 3. The graphical dependencies of the skidding and slipping coefficients $\delta_{20}, \delta_{10}$ on the values of the coefficient $k_{\delta}$ and the relative traction force $\psi$.

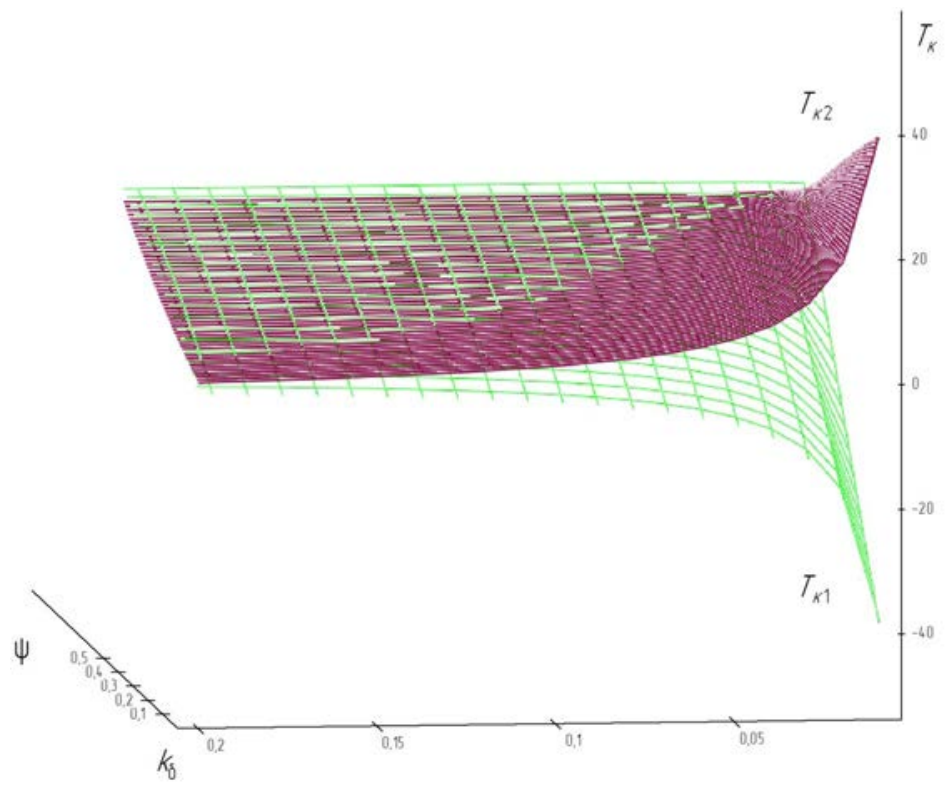

Fig. 4. The graphic dependences of traction (braking) forces of driving wheels on values of coefficient $k_{\delta}$ and relative traction force $\psi$.

Analysis of the presented graphical dependencies allows us to draw the following conclusions.

The difference between the slipping (skidding) of the lagging wheel and the slipping of the running one depends only on the value of the relative traction force $\psi$ and reaches the maximum value when $\psi=0$, i.e. in a transport mode. 
The values of the braking and traction forces of the driving wheels, on the contrary, almost do not depend on the relative traction force $\psi$ on loose ground support surfaces $\left(k_{\delta}>0,15\right)$, but they increase intensively with increasing traction properties of the driving surface $\left(k_{\delta}<0,1\right)$, especially in the zone of small values $\psi$ (transport and close to it modes of operation).

Significant additional traction and braking forces that occur in the wheel mover of an asymmetric balance rod with a blocked drive cause corresponding friction forces in the tire contact with the support surface and increased sliding in it due to increased slipping and skidding, which will cause additional wear of their tread.

Thus, the most unfavorable working conditions of the wheel mover of a traction machine with a blocked drive of an asymmetric balance rod will be when it moves without a traction load (transport mode) on a support surface with high traction properties.

Conclusion.

The algorithm of determination the parameters of wheel mover with a blocked asymmetrical balance bar drive of a traction machine has been developed. It takes into account the kinematic mismatch arising from the redistribution of the vertical reactions at the driving wheels due to off-center application of the pushing efforts based traction balance and equality of the actual speeds of the drive wheels at variable vertical load.

The most unfavorable working conditions of the wheel mover of a traction machine with asymmetric balance rod blocked drive causing increased tire wear and additional ("parasitic") loads on the drive will be when it moves on a support surface with high traction and coupling properties.

\section{References}

1. K.K. Shestopalov, Construction and Road Machines. Moscow, p. 383 (2008) (in Russian)

2. N.A. Ulyanov, The Theory of Self-propelled Wheeled Earth Moving Machines. Moscow, p. 520 (1969) (in Russian)

3. P.A. Sokol, Justification of the Parameters of the Asymmetric Balancing Drive of the Rear Driving Wheels of Forestry Vehicles: Abstract of Cand. Tech. Sciences Thesis. Voronezh, p. 16 (2019) (in Russian)

4. G.M. Kutkov, Tractors and Automobiles. Theory and Technological Properties. Moscow, p. 504 (2004) (in Russian)

5. Prof. Dipl.-lng. Jörnsen Reimpell, Fahrwerktechnik: Federung Fahrwerkmechanik. 2. Auflage. Vogel-Buchverlag Würyburg, p. 288 (1983)

6. Lng. Julius Mackerle, Automobil s lepši účinností. Praha (1985). SNTL Nakladatelstvi technicé literatury, p. 320 (1987)

7. E.A. Chudakov, Selected Works. Vol. 1. Car Theory. Moscow, p. 460 (1961) (in Russian)

8. V.A. Zhulay, A.A. Fedenev, D.I. Andrukhov, D.S. Peretrukhin, Determination of the Balance Bar's Suspension Axis Displacement of the of an Asymmetric Motor Grader Drive, High Technologies in Construction, No 2, pp. 113-118 (2019) (in Russian)

9. Y.M. Buzin, The Operation of the Grader's Balance Wheel Mover, Construction and Road Machines, No 11, pp. 46-51. (2014) (in Russian)

10. V.P. Lunevich, A.F. Kolyazin, D.Kh. Dachkovsky, V.A. Zhulay, L.Kh. Sharipov, On the Selection of Tires for Class 140 Motor Graders, Construction and Road Machines, No 6, pp. 14-16 (1995) (in Russian)

11. V.A. Zhulay, L.Kh. Sharipov, A.V. Skripchenkov, Justification of the Internal Combustion Engines for Graders Parameters, Construction and Road Machines, No 6, pp. 2-4 (2010) (in Russian) 